\title{
Editorial
}

\section{Inaugural editorial for pulmonary and critical care medicine}

\section{Yassine Amrani*}

Department of Infection, Immunity and Inflammation, University of Leicester, UK

It gives me great pleasure to launch this new journal entitled "Pulmonary and Critical Care Medicine" that is aimed at publishing novel findings addressing urgent and unmet clinical challenges in Respiratory Medicine. Pulmonary and Critical Care Medicine (PCCM) welcomes translational studies derived from multidisciplinary groups involving both basic scientists and clinicians who are working together to address aspects of pulmonary and critical care medicine ranging from disease susceptibility to disease pathogenesis to outcome prediction, and to heterogeneity of response to treatment and.

PCCM will be particularly interested in articles that describe ways to better improve patient classification into different subpopulations that is urgently needed for the development of improved personalized therapy and reduced health care costs in patients with complex lung disorders. PCCM will therefore prioritise articles that focus on the identification of novel biomarkers using "omics" approaches and their clinical validation and their utility in maximizing the efficacy of current and novel therapies. Investigators comparing the clinical impact of nonbiological computational and mathematical models of lung diseases are also encouraged to submit their work to Pulmonary and Critical Care Medicine.

Last but not least, more basic studies investigating lung diseases using in vitro biological models (as monolayers or 3D systems) derived from patient-derived lung structural cells such as airway smooth muscle, epithelial cells and fibroblasts will also be highly considered.
Copyright: (C)2016 Amrani Y. This is an open-access article distributed under the terms of the Creative Commons Attribution License, which permits unrestricted use, distribution, and reproduction in any medium, provided the original author and source are credited.
Correspondence to: Dr. Yassine Amrani, Department of Infection, Immunity and Inflammation, University of Leicester, National Institute for Health Research, Leicester Respiratory Biomedical Research Unit, Glenfield Hospital, Leicester, LE3 9QP, UK, Tel: +44 (0)116 2583694; E-mail: ya26@le.ac.uk

Received: March 20, 2016; Accepted: March 20, 2016; Published: March 24, 2016 